\title{
MedienPädagogik
}

Zeitschrift für Theorie und Praxis der Medienbildung

Themenheft Nr. 28: Tagungsband: Bildung gemeinsam verändern: Diskussionsbeiträge und Impulse aus Forschung und Praxis. Herausgegeben von David Meinhard, Valentin Dander, Andrea Gumpert, Christoph Rensing, Klaus Rummler und Timo van Treeck.

\section{[Wenn die Aussage lautet:] Spielerisch Geschichte lernen? [bezieht sich das worauf?]}

Tobias Winnerling

\begin{abstract}
Zusammenfassung
Digitale Spiele mit historischem Hintergrund sind eine populärkulturelle Remedialisierungsform der Bezugnahme auf 'Geschichte), die in der Geschichtswissenschaft immer noch umstritten ist. Für die Frage, ob historisches Lernen anhand von Videospielen sinnvoll möglich sein kann, bedürfen zwei Punkte besonderer Aufmerksamkeit. Erstens müssen die Geschichtswissenschaften sich in der Auseinandersetzung mit digitalen Spielen vor pauschalen Urteilen hinsichtlich der Repräsentationslogiken «des Historischen in diesem Medienformat hüten. Weder sind digitale Spiele hier prinzipiell andersartig als akademische Historiographie, noch können sie umstandslos an deren Massstäben gemessen werden. Zweitens muss eine präzise Definition sowohl des anzustrebenden didaktischen Nutzens eines Einsatzes von digitalen Spielen, als auch der Spielformate, die Möglichkeiten hierzu bieten können, erfolgen. Hierzu muss die Fokussierung der Debatte auf eine Dichotomie Blockbuster/Serious Games überwunden werden. Für einen Erfolg versprechenden didaktischen Einsatz von digitalen Spielen zur Geschichtsvermittlung müssen die Bezüge, die digitale Spiele auf den Referenzbereich 〈des Historischen〉 setzen, so gestaltet werden, dass sie über das Spiel hinausweisende Angebote machen, ohne ihre spielinterne Funktion zu destabilisieren.
\end{abstract}

[If the Statement Is:] Learning History by Playing? [This Refers to?]

\begin{abstract}
Digital games with historical background constitute a form of remedializing references to «history in popular culture that still is disputed by the historical sciences. The question «/s meaningful historical learning by playing digital games possible?» gives rise to two points of discussion to be addressed with care. First: in discussing digital games' representational logics of remedializing sthe historical the historical sciences need to distinguish carefully. Digital games are neither totally alien to the modes of historical academia in this respect, nor may they be judged according to academic standards wholesale. Second: there has to be a clear definition of the didactic goals to be achieved as well as of the types of games needed to achieve them. Focussing only on a dichotomy of blockbuster titles/serious games needs to be overcome. To make a didactically promising use of digital games in
\end{abstract}

Winnerling, Tobias. 2017. «[Wenn die Aussage lautet:] Spielerisch Geschichte lernen? [bezieht sich das worauf?]» MedienPädagogik 28, (22. Februar), 19-27. https://doi.org/10.21240/mpaed/28/2017.02.22.X. 
imparting historical knowledge possible, the relations established by those games in referencing sthe historicals have to be framed in a way that allows offering additional information without destabilizing their in-game functionality.

Männer wie Frauen, Alte wie Junge: Ein nicht zu unterschätzender Teil der Bevölkerung spielt gewohnheitsmässig digitale Spiele (BIU 2016, 30-33; 2011, 1f.). Darunter finden sich viele Titel und Serien mit Millionen verkaufter Exemplare und eindeutigen Bezügen auf Historisches - Assassin's Creed, Total War, Call of Duty, Sid Meier's Civilization, Anno, Hearts of Iron, Age of Empires, um nur einige zu nennen. Wenn Referenzen auf «Geschichte` in Spielen so beliebt sind, müssen sich Historiker/innen dann nicht damit auseinandersetzen? Und liesse sich Historie in (digitaler)spielerischer Form vielleicht sogar erfolgreich vermitteln (vgl. Kinkley 2009, 137)?

Auf das Risiko hin, dass das Experiment scheitert, sage ich zu beidem: Ja! Man muss es wenigstens versuchen. Aber während sich das leicht dahinschreibt, stehen diesem Vorhaben in der Praxis erhebliche Hürden entgegen. Mit zweien davon möchte ich mich auf der Grundlage eines mit Studierenden an der Heinrich-Heine-Universität Düsseldorf erarbeiteten Beispiels hier kurz näher auseinandersetzen. Dabei handelt es sich um das Projekt Lienzo, dessen Aufgabe es war, in enger Zusammenarbeit mit Studierenden ein (mit geringen finanziellen und technischen Mitteln erstelltes) digitales Spiel zu entwickeln, das einerseits als Spiel auch abseits jeder didaktischen Absicht funktioniert - und andererseits seinen Gegenstand in einer Form darstellt, die methodisch und theoretisch einer geschichtswissenschaftlichen Überprüfung standhielte (HHUD 2016).

\section{Interpretation: Vorsicht, Kategorienfehler!}

Mit der Remedialisierung eines Stoffs verbindet sich leicht die Vorstellung seiner völligen Neuordnung. Schliesslich gewinnen die Verweisketten, die den Referenzbereich abbilden, ihre Funktionalität nunmehr durch einen anderen Typ von Verknüpfungslogik: aus den Regeln, die das jeweilige Medium charakterisieren (vgl. Lyotard 1989, 10, 57f.). Die Darstellung von Geschichte in einem traditionellen linearen Medium wie dem gedruckten Buch unterscheidet sich auf den ersten Blick deutlich von der, die durch die interaktiven Schaltflächen und beweglichen Grafiken auf dem Bildschirm eines digitalen Spiels entsteht. Geschichtswissenschaftliche Bücher enthalten überwiegend Text - möglicherweise auch noch statische Bilder - und argumentieren intellektuell-demonstrativ. Digitale Spiele enthalten wenig Text, statische Bilder, bewegte Bilder, Audiosequenzen und Interaktionsmöglichkeiten; sie nutzen diese breite Palette medialer Darstellungsmittel für eine affektiv-persuasive Argumentationsweise. Aber das bedeutet nicht, dass die Regeln, die beide Formen der Medialisierungen 
des Referenzbereichs bestimmen, den wir als «die Geschichte) oder (das Historische) bezeichnen, deswegen notwendig so verschieden sind, wie ihre Anmutung suggeriert (Heinen 2011, 30). Das kann der Fall sein; es kann aber genauso gut lediglich ein Teilbereich des Regelsystems geändert oder neu hinzugefügt worden sein. Lienzo baut auf der - in Buchform vorliegenden - Darstellung eines konkreten historischen Ereignisses auf, der Eroberung Guatemalas durch den spanischen Conquistador Jorge de Alvarado und seine indigenen Truppen in den 1520er Jahren (Asselbergs 2004). Dieser Darstellung wiederum lag die Analyse eines indigenen mittelamerikanischen Geschichtsmediums zugrunde: Einer (textlosen) Wandkarte, des lienzo de Quauquechollan, mittlerweile volldigitalisiert durch die Universität Francisco Marroquín, Guatemala. Die piktographische Darstellung des lienzo de Quauquechollan lag in der schriftlichen Analyse bereits in remedialisierter Form vor, wäre in dieser Form also problemlos als klassisches Lehrmedium nutzbar. Aus der Feststellung, dass eine Remedialisierung vorliegt, darauf zu schliessen, diese neue Bezugsmöglichkeit auf den Referenzbereich sei von anderen soweit verschieden, dass deren Kategorien auf sie keine Anwendung finden könnten, geht also zu weit. Das bedeutet für Historiker/innen, dass sie sich nicht pauschal der Aufgabe verweigern können, sich auch mit den Bezügen auseinander zu setzen, die in digitalen Spielen - und über digitale Spiele - zum ‘Historischen` als Referenzbereich gebildet werden. In der Entwicklung von Lienzo haben wir uns die Freiheit genommen, das in positiver Weise als Ermutigung zu nutzen, offensiv vorzugehen. Es ging uns dabei darum, die dargestellten Inhalte in das Medium des Spiels zu übertragen, um sie besser vermittelbar zu machen. Die Spieler/innen werden in die Lage de Alvarados versetzt, und erzeugen durch ihre Aktionen als Conquistadoren eine eigene piktografische Repräsentation auf dem Bildschirm, die sich als eigener lienzo ausdrucken lässt.

Umgekehrt muss aber auch vermieden werden, diese Oberfläche als oberflächlich abzutun und die dort sichtbare Verschiedenheit der Regelsysteme der Geschichtswissenschaften einerseits und digitaler Spiele andererseits als unwesentlich für die Art und Weise der Bildung ihrer Aussagen zu bestimmen. Das kann mit der Vereinnahmung der Repräsentationen von «Geschichte), die digitale Spiele erzeugen, durch das Regelsystem der Geschichtswissenschaft enden (vgl. Montero Díaz und Paz Rebollo 2013, 163f.). In unserem Fall hiesse das, dass auf dem Bildschirm nichts anderes zu sehen sein dürfte als das, was sich faktisch gesichert der historischen Darstellung entnehmen lässt. Die Spieler/innen würden so zu reinen Ausführenden degradiert, die nur eine einzige, ‘historisch korrektes Repräsentation erzeugen könnten. Wir haben uns also für einen anderen Weg entschieden: Wir haben versucht, die der Situation der Conquista Guatemalas unterliegenden Prozesse im Spiel nachzumodellieren, aber damit eine von Beginn an fiktionalisierte Situation zu generieren. Das erlaubt es den Spieler/innen, in der Interaktion mit dem Programm eine Conquista zu führen und einen lienzo zu generieren, wie es sie hätte geben können, aber so 
nie gab. Das interaktive Nachvollziehen der Prozesse ersetzt also das Nachspielen des historisch verifizierbaren Ereignispfades. Es handelt sich bei den durch das Spiel generierten Repräsentationen also um unhistorische Szenarien, die aber dennoch beanspruchen, historische Erkenntnis vermitteln zu können. Was dann wiederum dazu führen kann, dass dergestaltige Repräsentationen von Historikern/-innen, die das Repräsentationssystem der Geschichtswissenschaften unreflektiert übertragen, zwangsläufig für falsch erklärt werden: Denn obwohl sie (vermeintlich) doch nach denselben Regeln gebildet wurden, sehen sie so verschieden aus! Da muss ein Fehler vorliegen - der dann bereitwillig auf der Seite des Spiels gefunden werden kann, ist es doch dieses, dessen Darstellung so nach der Diskrepanz zum als gesichert geltenden Faktenbestand als mehr oder weniger verfälscht gelesen werden kann (vgl. Schwarz 2015, 404f.). Ein solcher Authentizitätsfetischismus ist nicht nur bei Historikern/innen beliebt, sondern auch bei vielen historisch interessierten Spielern/innen - bis zum letzten Uniformknopf (vgl. Pöppinghege 2011, 463). Auch ich kann mich nicht davon freisprechen, zunächst derartige Urteile gefällt zu haben (vgl. Winnerling 2013, 727).

Der Widerstreit der beiden Darstellungsformen - konventionelle historische Repräsentation und digitale Spiele - kann jedoch zumindest entschärft werden, wenn eine vorsichtigere Annäherung betrieben wird. Wenn nicht sicher ist, ob und inwieweit die Regeln beider Systeme übereinstimmen, muss zunächst davon ausgegangen werden, dass das der Fall sein kann und sich wie bei jedem Remedialisierungsprozess nicht nur die Inhalte, sondern auch Teile der Logiken der älteren Medien in neuen wiederfinden lassen. Lyotards Mahnung eingedenk, dass Repräsentationen nicht intrinsisch wahr oder falsch sein können, sondern nur passend oder unpassend (vgl. 1989, 59), lässt sich die Debatte auch als Frage nach der Angemessenheit der jeweiligen Repräsentationen für spezifische Kontexte unter den Bedingungen ihres Mediums formulieren.

Das empfiehlt sich besonders bei der Frage nach dem Einsatz von digitalen Spielen in einem Kontext, in dem sie leicht prinzipiell unangemessen zu sein scheinen können: in der universitären geschichtswissenschaftlichen Lehre.

\section{Gegenstände: Was wäre ein passendes Spiel?}

Ein Medium didaktisch nutzen zu wollen, dessen Komplexität und Wirkungen wir noch immer nicht ganz verstehen, ist schwierig und vielleicht sogar nicht ganz ungefährlich. Inszeniert sich ein Videospiel historisch, könnte in einer Lernsituation nun das Missverständnis naheliegen, es träfe die Aussage, «Geschichte〉 habe sich zwar nicht exakt so abgespielt wie dargestellt, aber grundsätzlich so funktioniert wie gezeigt (vgl. Christesen und Machado 2010, 108f.) - auch wenn das bislang nur vermutet werden kann (vgl. Köck 2012, 11; Winnerling 2014, 159). 
Nichtsdestotrotz ist davon auszugehen, dass die Spieler/innen von digitalen Spielen, die historische Inhalte verarbeiten, irgendetwas während des Spielens lernen, und sei es nur, wie das Spiel funktioniert (vgl. Lampert et al. 2011, 118). Worum es gehen muss, ist zunächst herauszufinden, was und wie eigentlich spielerisch gelernt wird, und wie wir uns diese Prozesse zunutze machen können. Gerade beim Schwierigkeits- und Frustrationsmanagement sind schon herkömmliche digitale Spiele der konventionellenuniversitären Didaktik in den Geschichtswissenschaften weit voraus, ebenso bei der unkomplizierten und voraussetzungslosen Einübung von Grundfähigkeiten (vgl. ebd., 132f., 154f.).

Das oben skizzierte interpretatorische Problem hallt hier nach, denn fraglich ist dabei weiterhin: Wie trifft ein digitales Spiel eigentlich eine Aussage? (Und kann man überhaupt davon ausgehen, dass es so konstruiert ist, Aussagen treffen zu sollen?) Ein Text wie dieser fasst seine (also: meine) Aussagen in alphabetisch transkribierte Sätze. Sie sind verhältnismässig leicht reproduzierbar, lassen sich nachlesen, verstehen, und relativ problemlos mit nach denselben Regeln gebildeten Sätzen kritisieren. Digitale Spiele tun das nicht. Sie enthalten zuweilen derartige Sätze, aber diese bedienen zumeist andere Funktionen als die, eine Aussage über einen Referenzbereich zu generieren, der nicht das Spiel selbst ist, also Spieler/innen über im Spiel ablaufende Prozesse anzuweisen, auf solche aufmerksam zu machen, vor ihnen zu warnen oder über diese in ihrer ludischen, nicht referenziellen Funktion zu informieren. Wenn digitale Spiele historische Referenzen machen, so verwenden sie diese also meist in antonymischer Form, als Zitat, um eine Aussage über das Spiel selbst zu treffen und nicht über das, was der Inhalt des Zitates ist (vgl. Lyotard 1989, 67f.). Das trennt digitale Spiele als Medien deutlich von - beispielsweise - Filmen mit historischem Inhalt, denn der Referenzbereich derer Aussagen ist üblicherweise nicht der Film selbst, sondern (zumindest scheinbar) die erzählte «Geschichte). Während Nohr bereits überzeugend dargelegt hat, wie Aussagen von Videospielen einzelnen diskursiven Bereichen zugeordnet und darüber kontextualisiert werden können (vgl. 2014, 15), ist noch offen, woraus eine Aussage in einem Videospiel eigentlich besteht. Ein Analogieschluss erlaubt vielleicht eine erste Annäherung. Aussagen in Texten bestehen aus Sätzen, gegebenenfalls auch aus Bildern und Zeichen. Sätze werden aus Wörtern gebildet und können sowohl auf Bilder als auch auf Zeichen bezogen werden, um diese in eine konkrete Aussageform zu bringen. Alles, was das textuelle Medium abzubilden vermag, kann also Teil seiner Aussagen sein. Übertragen auf digitale Spiele hiesse das, dass wir auch hier damit rechnen müssen, dass alles, was das Spiel abzubilden vermag - Text, Bild, Ton, Animation, Interaktion - Bestandteil seiner Aussagen sein kann. Für Bestandteile, die historische Bezüge nutzen, bedeutet das, dass sie - sicherlich nicht bewusst - wahrscheinlich auch zur impliziten Konstruktion historischer Aussagen rezipiert werden (vgl. Schut 2007, 218). Solange es sich um Titel handelt, die keinen Anspruch auf historische Korrektheit erheben, ist das 
wenig problematisch. Wenn aber versucht wird, ein digitales Spiel bewusst mit diesem Anspruch auszustatten, um es auch geschichtsdidaktisch einsetzen zu können, muss besondere Sorgfalt darauf verwendet werden, die Möglichkeiten zur Konstitution historisch falscher oder missverständlicher Aussagen einzuschränken und die dafür notwendigen Reflexionsinstrumente zur Verfügung zu stellen. Da Spiele aufgrund des breiteren Spektrums vorgesehener menschlicher Interaktionsmöglichkeiten weniger strikt formuliert sind als Texte, dürften auch die aus innen erschliessbaren Aussagen weniger leicht reproduzierbar und nachvollziehbar sein als meine Sätze in diesem Text. Über ihre Verständlichkeit ist damit noch nichts gesagt; was aber die Möglichkeit zur adäquaten Kritik angeht, so ist zumindest klar, dass sie nicht problemlos im selben Medium vollzogen werden kann. Text als Medium zur Konstruktion historischer Aussagen lässt sich im selben Medium kritisch betrachten. Alle übrigen audiovisuellen Medien, die dazu vorgesehen sind, linear rezipiert zu werden, lassen sich relativ problemlos in - ebenfalls linear operierender - textueller Form wiedergeben. Schliesslich ist kaum zu verlangen und zu erwarten, dass Studierende digitale Spiele programmieren, nur um Aussagen über andere digitale Spiele treffen zu können. Klar ist auch, dass die vielfältigen Aussagen, die durch die Spieler-Spiel-Interaktion gebildet werden können, sich für die Integration in ein lineares Lernkonzept nicht gut anbieten. Der geringe kommerzielle Erfolg von reinen Lernspielen, «Serious Games), mag auch hierauf zurückzuführen sein. Und selbst Blockbuster-Titel verlieren stark an Reiz, sobald sie als primär didaktische Instrumente Verwendung finden (vgl. Charsky und Ressler 2011, 611; McMichael 2007, 213).

Um ein geschichtswissenschaftlich didaktisch brauchbares digitales Spiel entwickeln zu können, müssen wir also nicht vom Lernen, sondern vom Spielen her denken (vgl. Heinen 2011, 28). Wir brauchen Spiele, die ein wissenschaftlich fundiertes, über das Spiel hinausgehendes Angebot machen, dieses aber nicht als eigentlichen Spielzweck über, sondern als Mitnahmeeffekt neben das Spielen setzen. Unser im Rahmen eines Lehr- und Forschungsprojekts zur Zeit in der Entwicklung befindliche Titel Lienzo stellt einen ersten Feldversuch in dieser Richtung dar. Wir wollen in diesem Projekt sicherstellen, dass das Spiel Aussagen, die wir als Aussagen über einen als historisch definierten Referenzbereich verstanden wissen möchten, in geschichtswissenschaftlich wohlgeformter Art trifft, wissen aber im Voraus nicht, aus welchen Elementen diese Aussagen sich jeweils zusammensetzen werden. Uns bleibt also nur, die einzelnen Elemente so zu gestalten, dass wir bei ihnen mit hinreichender Sicherheit davon ausgehen können, dass sie der historischen Kritik zugänglich sind, wenn nicht sogar ihr genügen. Wir legen also unsere Quellen offen und stellen die wichtigsten Informationen zum Verständnis der aus dem Spiel auf die «Geschichte> zielenden Referenzen im Spiel selbst gebündelt zur Verfügung.

Zugleich bedeutet aber dieser Zugewinn an historisch-kritischer Genauigkeit auf der Ebene der Einzelelemente einen Verzicht des Spielgeschehens auf einen konkreten 
Referenzcharakter. Es geht eben nicht um den Nachvollzug der faktischen Ereignisse der Conquista Guatemalas, sondern darum, in fiktionalisierten Szenarien die Prozesse verstehen zu lernen, die der historischen Situation unterlagen (soweit wir sie nachbilden konnten). Die Interaktionsfreiheit der Spieler/innen - die in einem modellhaft geregelten Rahmen die Interaktionsmöglichkeiten zeitgenössischer Akteure/innen nachzeichnen soll - macht es, anders als in anderen ähnlichen experimentellen digitalen Spielen wie Ubisofts Valiant Hearts (Schwarz 2015, 428-30), unmöglich, die einzelnen Spielverläufe als Nacherzählungen einer historischen Wahrheit, eines konkreten Ereignisses zu konstruieren (vgl. Deleuze und Guattari 2013, 163). Vielmehr handelt es sich um fiktionalisierte Projektionen eines Möglichkeitsraums: Gegeben, folgende Elemente hätten in der historischen Situation in der Konfiguration vorgelegen, in der sie beim Spielstart angeordnet wurden, so wäre folgendes Muster aktiver Verknüpfungen durch Handlung sinnvoll und möglich gewesen - der jeweilige Spielverlauf. «Auf diese Weise provoziert die historische Erkenntnis selbst eine Vielzahl von Bedeutungen / (Hypothesen, Deutungen), um sie durch das Sieb der Beweisführung zu passieren.» (Lyotard 1989, 91) Ob wir aber am Ende dazu wirklich in der Lage sein werden - und welche Erkenntnisse damit verbunden sein werden - bleibt abzuwarten; dieses Spiel ist noch nicht ausgespielt.

\section{Literatur}

Asselbergs, Florine. 2004. Conquered conquistadors: the Lienzo de Quauhquechollan. A Nahua vision of the conquest of Guatemala. Leiden: CNWS publications.

BIU. 2016. Jahresreport der Computer- und Videospielebranche in Deutschland 2016. Berlin.

BIU. 2011. Gamer in Deutschland. Eine Studie des Bundesverband Interaktive Unterhaltungssoftware e. V. (BIU) auf Basis einer Befragung von 25.000 Deutschen durch die GfK. Berlin.

Charsky, Dennis, und William Ressler. 2011. «Games are made for fun:> Lessons on the effects of concept maps in the classroom use of computer games.» Computers \& Education 56:604-615.

Christesen, Paul, und Dominic Machado. 2010. «Video Games and Classical Antiquity.» Classical World 104 (1):107-110.

Deleuze, Gilles, und Felix Guattari. 2013. A Thousand Plateaus. Capitalism and Schizophrenia, übers. v. Brian Massumi. London u.a.: Bloomsbury.

Heinen, Armin. 2011. «Mediaspektion der Historiographie. Zur Geschichte der Geschichtswissenschaft aus medien- und technikgeschichtlicher Perspektive.» Zeitenblicke 10 (1):Abs. 1-43. http://www.zeitenblicke.de/2011/1/Heinen/index_html, URN:nbn:de:0009-9-30184.

Kinkley, Jonathan. 2009. «Art Thief. An Educational Computer Game Model of Art Historical Instruction.» Leonardo 42 (2):133-137. 
Köck, Julian. 2012. "Geschichtsbilder im Weltkriegsshooter und ihre Rezeption beim Spieler.» Zeitschrift für digitale Geschichtswissenschaften 1:1-22. http://universaar.uni-saarland.de/ journals/index.php/zdg/article/view/296.

Lampert, Claudia, Christiane Schwinge, und Sheela Teredesai. 2011. "Kompetenzförderung in und durch Computerspiele(n).» In Kompetenzen und exzessive Nutzung bei Computerspielern: Gefordert, gefördert, gefährdet, Medienforschung der Landesanstalt für Medien Nordrhein-Westfalen 66, herausgegeben von Jürgen Fritz, Claudia Lampert, Jan-Hinrik Schmidt, Tanja Witting, 117-181. Düsseldorf: Landesanstalt für Medien.

Lienzo de Quauquechollan, http://www.lienzo.ufm.edu/ [Zuletzt besucht: 14. November 2016].

HHUD. 2016. Lienzo, https://www.uni-duesseldorf.de/redaktion/index.php?id=84163 [Zuletzt besucht: 03. August 2016].

Lyotard, Jean-François. 1989. Der Widerstreit, übers. v. Jürgen Vogel. München: Wilhelm Fink.

McMichael Andrew. 2007. «PC Games and the Teaching of History.» The History Teacher 40 (2):203-218.

Montero Díaz, Julio, und María Antonia Paz Rebollo. 2013. «Historia audiovisual para una sociedad audiovisual.» Historia Critica 49:159-183.

Nohr, Rolf. 2014. «The Game is a Medium: The Game is a Message.»In Early Modernity and Video Games, herausgegeben von Tobias Winnerling, Florian Kerschbaumer, 2-23. Newcastleupon-Tyne: Cambridge Scholars Publishing.

Pöppinghege, Rainer 2011. «Pedanterie im Cyberspace.» Geschichte in Wissenschaft und Unterricht 62: 459-468.

Schut, Kevin. 2007. «Strategic Simulations and Our Past. The Bias of Computer Games in the Presentation of History.» Games and Culture 2 (3):213-235.

Schwarz, Angela. 2015. «Game Studies und Geschichtswissenschaft.»In Game Studies. Aktuelle Ansätze der Computerspielforschung, herausgegeben von Klaus Sachs-Hombach, Jan-Noël Thon, 398-447. Magdeburg: Herbert von Halem.

Winnerling, Tobias. 2013. «Sicherer Berg, gefährlicher Feind. Natürlicher und militärischer Raum im Computerspiel zur Frühen Neuzeit.» In Sicherheit in der Frühen Neuzeit. Norm Praxis - Repräsentation, herausgegeben von Christoph Kampmann und Ulrich Niggemann, 712-727. Köln/Weimar/Wien: Böhlau.

Winnerling, Tobias. 2014. «The eternal recurrence of all bits. How historicizing video games' series transform factual history into affective historicity.» eludamos. Journal for Computer Game Culture, 8:129-149. Special Issue Digital Seriality.

\section{Ludografie}

Age of Empires-Serie: Age of Empires. 1997. Ensemble Studios [Mac OS, Microsoft Windows] Microsoft; Age of Empires II: The Age of Kings. 2005. Ensemble Studios [Mac OS, Microsoft Windows, Playstation 2] Microsoft; Age of Empires III. 1999. Ensemble Studios [Mac OS, Microsoft Windows] Microsoft; Age of Empires Online. 2011-2014. Gas Powered Games/Robot Entertainment [Microsoft Windows] Microsoft. 
Anno-Serie (historisch inszenierte Titel): Anno 1602. 1998. Max Design/Sunflowers [Microsoft Windows] Infogrames; Anno 1503. 2002. Max Design [Microsoft Windows] Sunflowers; Anno 1701. 2006. Related Designs [Microsoft Windows, Nintendo DS, Smartphone] Aspyr/KochMedia/Sunflowers: Anno 1404. 2009. Related Designs/Blue Byte [Microsoft Windows] Ubisoft.

Assassin's Creed-Serie (Haupttitel): Assassin's Creed. 2007. Ubisoft [Mac OS, Microsoft Windows, PlayStation3; Xbox360] Ubisoft; Assassin's Creed II. 2009. Ubisoft [Microsoft Windows, Nintendo WiiU PlayStation3; Smartphone; Xbox360] Ubisoft; Assassin's Creed III. 2012. Ubisoft [Microsoft Windows, Nintendo WiiU PlayStation 3; Xbox360] Ubisoft; Assassin's Creed IV: Black Flag. 2013. Ubisoft [Microsoft Windows, Nintendo WiiU PlayStation 3 u. 4; Xbox360 u. One] Ubisoft; Assassin's Creed Unity. 2014. Ubisoft [Microsoft Windows, PlayStation 4, Xbox One] Ubisoft; Assassin's Creed Syndicate. 2015. Ubisoft [Microsoft Windows, PlayStation 4, Xbox One] Ubisoft.

Call Of Duty-Serie (historisch inszenierte Titel): Call of Duty. 2003. Infinity Ward [Mac OS X, Microsoft Windows, Nokia N-Gage, PlayStation 3, Xbox 360] Activision/Aspyr; Call of Duty 2. 2006. Infinity Ward [Mac OS X, Microsoft Windows, Xbox 360] Activision; Call of Duty 3. 2006. Treyarch [Nintendo Wii, Playstation 2, Xbox, Xbox 360] Activision; Call of Duty: World at War. 2008. Treyarch [Microsoft Windows, Nintendo DS u. Wii, PlayStation 2, PlayStation 3, Xbox 360] Activision; Call of Duty: Black Ops. 2010. Treyarch/IdeaWorks/n-Space [Mac OS X, Microsoft Windows, Xbox 360, Xbox One, PlayStation 3, Nintendo Wii] Activision.

Civilization-Serie (Haupttitel): Sid Meier's Civilization. 1991. Microprose [Amiga, Atari ST, MSDOS, Apple Macintosh, SNES, Microsoft Windows, N-Gage]; Sid Meier's Civilization II. 1996. Microprose [Mac OS, Microsoft Windows, PlayStation]; Sid Meier's Civilization III. 2001. Firaxis [Mac OS, Microsoft Windows] Infogrames; Sid Meier's Civilization IV. 2005. Firaxis [Mac OS, Microsoft Windows] 2K Games; Sid Meier's Civilization V. 2010. Firaxis [Linux, Mac OS, Microsoft Windows] 2K Games; Sid Meier's Civilization VI. 2016. Firaxis [Linux, Mac OS, Microsoft Windows] 2K Games.

Hearts of Iron-Serie: Hearts of Iron. 2002. Paradox Development Studio [Mac OS, Microsoft Windows] Strategy First; Hearts of Iron II. 2005. Paradox Development Studio [Mac OS, Microsoft Windows] Paradox Interactive; Hearts of Iron III. 2009. Paradox Development Studio [Mac OS, Microsoft Windows] Paradox Interactive; Hearts of Iron IV. 2016. Paradox Development Studio [Mac OS, Microsoft Windows] Paradox Interactive.

Total War-Serie (Haupttitel): Shogun: Total War. 2000. Creative Assembly [Microsoft Windows] Electronic Arts/Sega; Medieval: Total War. 2002. Creative Assembly [Microsoft Windows] Activision; Rome: Total War. 2004. Creative Assembly [iOS, Mac OS, Microsoft Windows] Activision/Sega; Medieval II: Total War. 2006. Creative Assembly [Linux, Mac OS, Microsoft Windows] Sega; Empire: Total War. 2009. Creative Assembly/Feral Interactive [Linux, Mac OS, Microsoft Windows] Sega; Napoleon: Total War. 2010. Creative Assembly/Feral Interactive [Mac OS, Microsoft Windows] Sega; Total War: Shogun II. 2011. Creative Assembly [Mac OS, Microsoft Windows] Sega; Total War: Rome II. 2013. Creative Assembly [Mac OS, Microsoft Windows] Sega; Total War: Attila. 2015. Creative Assembly [Microsoft Windows] Sega. 